\title{
Odd Hyperbolic Cosine Exponential-Exponential (OHC-EE) Distribution
}

\author{
Omid Kharazmi $^{1}$ - Ali Saadatinik ${ }^{2} \cdot$ Shahla Jahangard ${ }^{3}$ \\ Received: 18 December 2018 / Revised: 11 March 2019 / Accepted: 25 March 2019/ \\ Published online: 17 May 2019 \\ (๑) The Author(s) 2019
}

\begin{abstract}
In the present paper, we introduce a new lifetime distribution based on the general odd hyperbolic cosine-FG model. Some important properties of proposed model including survival function, quantile function, hazard function, order statistic are obtained. In addition estimating unknown parameters of this model will be examined from the perspective of classic and Bayesian statistics. Moreover, an example of real data set is studied; point and interval estimations of all parameters are obtained by maximum likelihood, bootstrap (parametric and non-parametric) and Bayesian procedures. Finally, the superiority of proposed model in terms of parent exponential distribution over other fundamental statistical distributions is shown via the example of real observations.
\end{abstract}

Keywords Lifetime distribution · Hazard rate $\cdot$ Bayesian method

\section{Introduction}

Alzaatreh et al. [2] have introduced a new model of lifetime distributions, which the researchers refer to its especial case as odd- $G$ distribution. It is based on the combination of an arbitrary cumulative distribution function $C D F$ with odd ratio of the baseline $C D F$ as $G$. The integration form of the new $C D F$ is

$$
H(x)=\int_{-\infty}^{\frac{G(x)}{1-G(x)}} f(t) d t,
$$

\footnotetext{
$凶$ Omid Kharazmi

omid.kharazmi@vru.ac.ir

Ali Saadatinik

a.saadatinik@stu.umz.ac.ir

1 Department of Statistics, Faculty of Sciences, Vali-e-Asr university of Rafsanjan, Rafsanjan, Iran

2 Department of Statistics, University of Mazandaran, Babolsar, Iran

3 Department of Statistics, Isfahan Univesity of Technology, Isfahan, Iran
} 
where $f$ is the probability density function $P D F$ of arbitrary $C D F$. This interesting method attracted the attention of some researchers. We refer the reader to $[1,4]$. Generating new model based on this method resulted in creating very flexible statistical model. Recently, Kharazmi et al. [7] have introduced a new general family of lifetime distribution based on the odd ratio of a parent distribution $G$ for general hyperbolic cosine-F ( $H C F$ ) family of lifetime distributions that recently proposed by Kharazmi and Saadatinik [6]. This new model will be denoted by odd-HCF-G (or OHCFG) distribution.

In the present paper, we introduce a special case of the odd-HCF-G model by applying two exponential distributions instead of $F$ and $G$ models.It is referred to as $O H C E E$ distribution. One of our main motivation to introduce this new distribution is to provide more flexibility for fitting real datasets compare to other well-known classic statistical distributions.

We provide a comprehensive discussion about statistical and reliability properties of new $O H C E E$ model. Furthermore, we consider maximum likelihood and bootstrap estimation procedures in order to estimate the unknown parameters of the new model for a complete data set. In addition, parametric and non-parametric bootstrap confidence intervals are calculated.

The rest of the paper organized as follows. In the Sect. 2, we review the main statistical and reliability properties of recently proposed model by Kharazmi et al. [7]. In Sect. 3, by considering the two exponential distributions as the base distributions, a new model is presented according to the general model discussed in Sect. 1 and its prominent characteristics are studied. This new model refer to as $O H C E E$ distribution. In Sect. 4, we examine the inferential procedures for estimation unknown parameters of the $O H C E E$ model. In this Section, we provide discussions about the maximum likelihood, Bayesian and bootstrap procedures. Applications and numerical analysis of an real data set are presented in Sect. 5. Finally, in Sect. 6 the paper is concluded.

\section{Preliminaries of HCF and OHCFG Lifetime Models}

In this section, we review the structure of two general $H C F$ and $O H C F G$ models. Kharazmi and Saadatinik [6] introduced a family of distributions using hyperbolic c function. The hyperbolic cosine has similar name to the trigonometric functions, but it is defined in terms of the exponential function as follows:

$$
\cosh (x)=\frac{e^{x}+e^{-x}}{2}
$$

According to Kharazmi and Saadatinik [6] a random variable $\mathrm{X}$ has a hyperbolic cosine-F (HCF) distribution if its cumulative distribution function (cdf) is given by

$$
G(x)=\frac{2 e^{a}}{e^{2 a}-1} \sinh (a F(x)),
$$

where $x>0, a>0$. 
Motivated by idea of Alzaatreh et al. [2], a new class of statistical distributions have proposed by Kharazmi et al [7]. The new model is constructed by applying Alzaattreh idea to the hyperbolic cosine-F ( $H C F$ ) family of lifetime distributions.

The CDF of new model is defined as

$$
\begin{aligned}
H(x) & =\int_{0}^{\frac{G(x)}{1-G(x)}} \frac{2 a e^{a}}{e^{2 a}-1} f(t) \cosh (a F(t)) \mathrm{d} t \\
& =\frac{2 e^{a}}{e^{2 a}-1} \sinh \left(a F\left(\frac{G(x)}{1-G(x)}\right)\right)
\end{aligned}
$$

The density of OHC-FG model can be obtained as

$$
h(x)=\frac{2 a e^{a}}{e^{2 a}-1} \frac{g(x)}{(1-G(x))^{2}} f\left(\frac{G(x)}{1-G(x)}\right) \cosh \left(a F\left(\frac{G(x)}{1-G(x)}\right)\right)
$$

The survival reliability $\bar{H}(x)$ and the hazard rate function $r(x)$ for OHC-FG distribution are in the following form

$$
\bar{H}(x)=1-\frac{2 e^{a}}{e^{2 a}-1} \sinh \left(a F\left(\frac{G(x)}{1-G(x)}\right)\right)
$$

and

$$
r(x)=\frac{\frac{2 a e^{a}}{e^{2 a}-1} \frac{g(x)}{(1-G(x))^{2}} f\left(\frac{G(x)}{1-G(x)}\right) \cosh \left(a F\left(\frac{G(x)}{1-G(x)}\right)\right)}{1-\frac{2 e^{a}}{e^{2 a}-1} \sinh \left(a F\left(\frac{G(x)}{1-G(x)}\right)\right)},
$$

respectively. The $p$ th quantile $x_{p}$ of the OHC-FG distribution can be obtained as

$$
x_{p}=G^{-1}\left(\frac{F^{-1}\left(\frac{1}{a} \operatorname{arcsinh}\left(\frac{e^{2 a}-1}{2 e^{a}} p\right)\right)}{1+F^{-1}\left(\frac{1}{a} \operatorname{arcsinh}\left(\frac{e^{2 a}-1}{2 e^{a}} p\right)\right)}\right) ; \quad 0 \leq p \leq 1 .
$$

$\operatorname{arcsinh}(x)=\ln \left(x+\sqrt{x^{2}+1}\right)$, then we get Since

$$
x_{p}=G^{-1}\left(\frac{F^{-1}\left(\frac{1}{a} \log \left(\frac{e^{2 a}-1}{2 e^{a}} p+\sqrt{\left.\left(\frac{e^{2 a}-1}{2 e^{a}} p\right)^{2}+1\right)}\right)\right.}{1+F^{-1}\left(\frac{1}{a} \log \left(\frac{e^{2 a}-1}{2 e^{a}} p+\sqrt{\left(\frac{e^{2 a}-1}{2 e^{a}} p\right)^{2}+1}\right)\right)}\right) .
$$

Hence, If the baseline $\mathrm{F}$ and $\mathrm{G}$ distributions are invertable then we can easily generate random samples from the OHC-FG distribution. 


\section{An Especial Case of OHCFG Model}

we apply the OHC-FG method to a specific class of distribution functions, namely to an exponential distribution and call this new distribution, three-parameter OHC-EE distribution.

Definition A random variable $\mathrm{X}$ has $\operatorname{OHC}-\operatorname{EE}\left(a, \lambda_{1}, \lambda_{2}\right)$, if its probability density function (PDF) is given by

$$
\begin{aligned}
h(x) & =\frac{2 a e^{a}}{e^{2 a}-1} \lambda_{1} e^{\lambda_{1} x} \lambda_{2} e^{-\lambda_{2}\left(e^{\lambda_{1} x}-1\right)} \cosh \left(a\left(1-e^{-\lambda_{2}\left(e^{\lambda_{1} x}-1\right)}\right)\right) ; \\
x & >0, a, \lambda_{1}, \lambda_{2}>0 .
\end{aligned}
$$

The CDF of (4) can be written as

$$
H(x)=\frac{2 e^{a}}{e^{2 a}-1} \sinh \left(a\left(1-e^{-\lambda_{2}\left(e^{\lambda_{1} x}-1\right)}\right)\right),
$$

also, survival and hazard rate functions are given by

$$
\bar{H}(x)=1-\frac{2 e^{a}}{e^{2 a}-1} \sinh \left(a\left(1-e^{-\lambda_{2}\left(e^{\lambda_{1} x}-1\right)}\right)\right),
$$

and the hazard rate function is given by

$$
r(x)=\frac{h(x)}{\bar{H}(x)}=\frac{2 a e^{a} \lambda_{1} e^{\lambda_{1} x} \lambda_{2} e^{-\lambda_{2}\left(e^{\lambda_{1} x}-1\right)} \cosh \left(a\left(1-e^{-\lambda_{2}\left(e^{\lambda_{1} x}-1\right)}\right)\right)}{e^{2 a}-1-2 e^{a} \sinh \left(a\left(1-e^{-\lambda_{2}\left(e^{\lambda_{1} x}-1\right)}\right)\right)}
$$

\subsection{Some Properties of the OHCEE Distribution}

In this section, we obtain some properties of the OHCEE distribution, involving quantiles, moments, moment generating function and extreme order statistics Some shapes of density function and hazard rate function are shown in Figs. 1 and 2.

\subsection{Quantiles}

For the OHCEE distribution, the $p$ th quantile $x_{p}$ is the solution of $H\left(x_{p}\right)=p$, hence

$$
\begin{aligned}
x_{p} & =\frac{1}{\lambda_{1}} \log \left(1-\frac{1}{\lambda_{2}} \log \left(1-\frac{1}{a} \operatorname{arcsinh}\left(\frac{e^{2 a}-1}{2 e^{a}} p\right)\right)\right) \\
& =\frac{1}{\lambda_{1}} \log \left(1-\frac{1}{\lambda_{2}} \log \left(1-\frac{1}{a} \log \left(\frac{e^{2 a}-1}{2 e^{a}} p+\sqrt{\left(\frac{e^{2 a}-1}{2 e^{a}} p\right)^{2}+1}\right)\right) ; 0 \leq p \leq 1\right.
\end{aligned}
$$

which is the base of generating OHCEE random variates. 

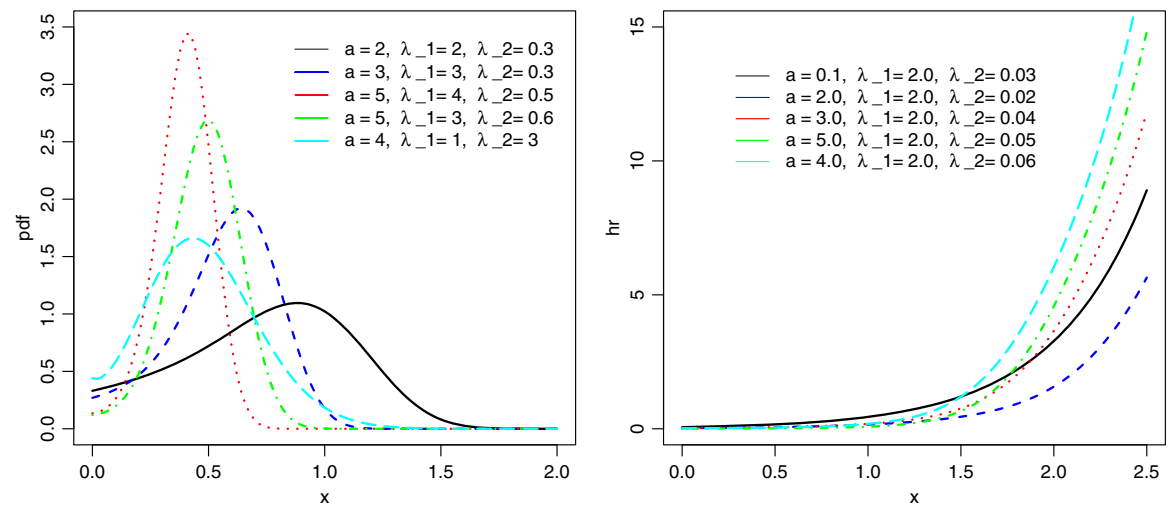

Fig. 1 plots of the $\operatorname{OHC}-\operatorname{EE}\left(a, \lambda_{1}, \lambda_{2}\right)$ (left) and failure rate function (right) for selected values of a, $\lambda_{1}, \lambda_{2}$
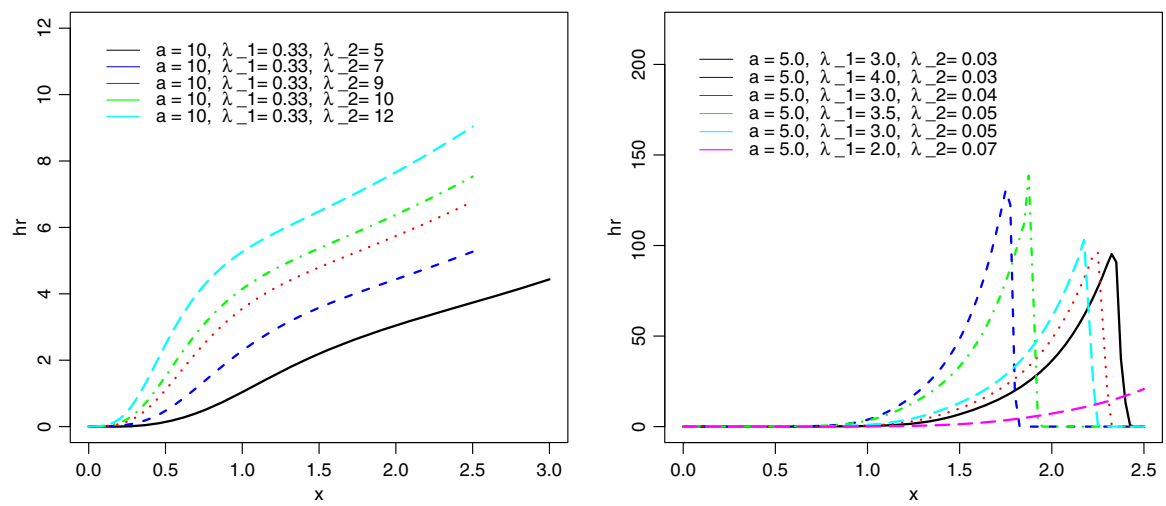

Fig. 2 failure rate function shapes for selected values of the parameters

\subsection{Moments and Moment Generating Function}

In this subsection, moments and related measures including coefficients of variation, skewness and kurtosis are presented. Tables of values for the first six moments, standard deviation (SD), coefficient of variation (CV), coefficient of skewness (CS) and coefficient of kurtosis $(\mathrm{CK})$ are also presented. The rth moment of the OHCEE distribution, denoted by $\mu_{r}^{\prime}$ is

$$
\begin{aligned}
\mu_{r}^{\prime} & =E\left(X^{r}\right)=\frac{2 a e^{a}}{e^{2 a}-1} \int_{0}^{\infty} x^{r} \lambda_{1} e^{\lambda_{1} x} \lambda_{2} e^{-\lambda_{2}\left(e^{\lambda_{1} x}-1\right)} \sum_{n=0}^{\infty} \frac{a^{2 n}\left(1-e^{-\lambda_{2}\left(e^{\lambda_{1} x}-1\right)}\right)^{2 n}}{(2 n) !} \mathrm{d} x \\
& =\sum_{n=0}^{\infty} \frac{a^{2 n+1}}{(2 n) !} \frac{2 e^{a}}{e^{2 a}-1} \int_{0}^{\infty} x^{r} \lambda_{1} e^{\lambda_{1} x} \lambda_{2} e^{-\lambda_{2}\left(e^{\lambda_{1} x}-1\right)}\left(1-e^{-\lambda_{2}\left(e^{\lambda_{1} x}-1\right)}\right)^{2 n} \mathrm{~d} x \\
& =\sum_{n=0}^{\infty} \frac{a^{2 n+1}}{(2 n) !} \frac{2 e^{a}}{e^{2 a}-1} \int_{0}^{\infty}\left(\frac{1}{\lambda_{1}} \log (1+y)\right)^{r} \lambda_{2} e^{-\lambda_{2} y}\left(1-e^{-\lambda_{2} y}\right)^{2 n} \mathrm{~d} y
\end{aligned}
$$


OHCEE(5, lambda1, lambda2)

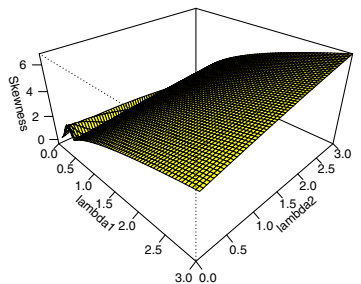

$\operatorname{OHCEE}(5$, lambda1, lambda2)

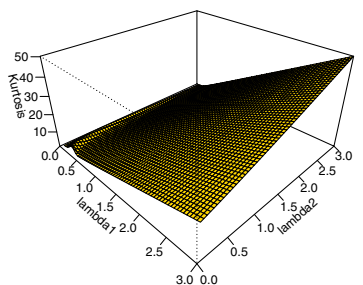

$\operatorname{OHCEE}(5$, lambda1, lambda2)

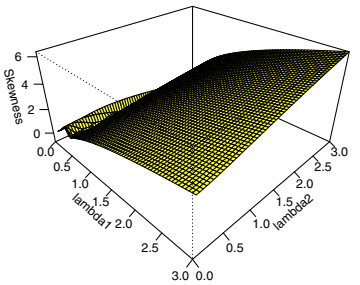

$\operatorname{OHCEE}(2.5$, lambda1, lambda2)

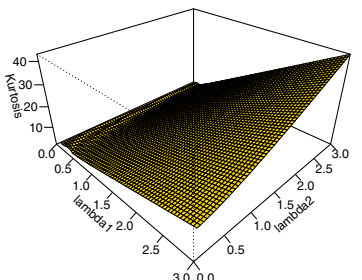

OHCEE(0.2, lambda1, lambda2)

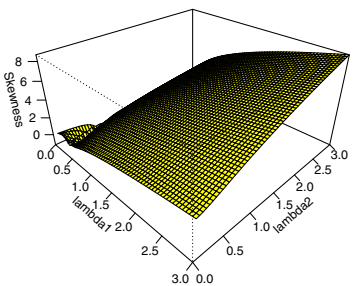

OHCEE(0.2, lambda1, lambda2)

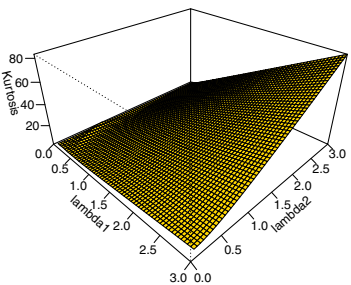

Fig. 3 3D plots of skewness and kurtosis of OHCEE distribution for some fixed values of parameter $a$

$$
\begin{aligned}
= & \sum_{n=0}^{\infty} \frac{a^{2 n}}{\lambda_{1}^{r}(2 n+1) !} \frac{2 e^{a}}{e^{2 a}-1} \int_{0}^{\infty}(\log (1+y))^{r}(2 n+1) \lambda_{2} e^{-\lambda_{2} y}\left(1-e^{-\lambda_{2} y}\right)^{2 n} \mathrm{~d} y \\
= & \sum_{n=0}^{\infty} \frac{a^{2 n}}{\lambda_{1}^{r}(2 n+1) !} \frac{2 e^{a}}{e^{2 a}-1} \int_{0}^{\infty}(\log (1+y))^{r} f_{G E\left(2 n+1, \lambda_{2}\right)}(y) \mathrm{d} y \\
= & \sum_{j=1}^{\infty} \sum_{n=0}^{\infty} \frac{d_{r . j}(-1)^{j} a^{2 n}}{\lambda_{1}^{r}(2 n+1) !} \frac{2 e^{a}}{e^{2 a}-1} \int_{0}^{\infty} y^{j} f_{G E\left(2 n+1, \lambda_{2}\right)}(y) \mathrm{d} y \\
= & \sum_{j=1}^{\infty} \sum_{n=0}^{\infty} \frac{d_{r . j}(-1)^{j} a^{2 n}}{\lambda_{1}^{r}(2 n+1) !} \frac{2 e^{a}}{e^{2 a}-1} E_{X_{G E\left(2 n+1, \lambda_{2}\right)}\left[Y^{j}\right]}
\end{aligned}
$$

where GE is Generalized Exponential distribution. The above integrate follows by using the series

$$
\log ^{r}(1+y)=\left(-\sum_{k=1}^{\infty} \frac{(-1)^{k}}{k} y^{k}\right)^{r}
$$

see the website https://www.wolframalpha.com, and by using the identity

$$
\left(\sum_{j=0}^{\infty} a_{j} x^{j}\right)^{t}=\sum_{j=0}^{\infty} d_{t . j} x^{j}
$$

where $d_{t . j}=\left(j a_{0}\right)^{-1} \sum_{m=1}^{j}[m(t+1)-j] a_{m} d_{t . j-m}$ and $d_{t .0}=a_{0}^{t}$. 
Table 1 Moments of the OHCEE distribution for some parameter values a $=2$

\begin{tabular}{lllll}
\hline$\mu_{r}^{\prime}$ & $\lambda_{1}=0.5, \lambda_{2}=0.5$ & $\lambda_{1}=0.5, \lambda_{2}=1.5$ & $\lambda_{1}=1.5, \lambda_{2}=0.5$ & $\lambda_{1}=1.5, \lambda_{2}=1.5$ \\
\hline$\mu_{1}^{\prime}$ & 2.333524 & 1.185911 & 0.7778413 & 0.3953037 \\
$\mu_{2}^{\prime}$ & 6.861136 & 1.943436 & 0.7623485 & 0.2159373 \\
$\mu_{3}^{\prime}$ & 22.67089 & 3.760179 & 0.8396628 & 0.1392659 \\
$\mu_{4}^{\prime}$ & 81.05204 & 8.144519 & 1.000642 & 0.1005496 \\
$\mu_{5}^{\prime}$ & 307.6889 & 19.22541 & 1.266209 & 0.0791169 \\
$\mu_{6}^{\prime}$ & 1226.292 & 48.65189 & 1.682157 & 0.0667378 \\
$\mathrm{SD}$ & 1.415802 & 0.537051 & 0.1573114 & 0.0596723 \\
$\mathrm{CV}$ & 0.6067227 & 0.4528594 & 0.3953037 & 0.1509531 \\
$\mathrm{CS}$ & 0.0312526 & 0.4615376 & 0.0312529 & 0.4615372 \\
$\mathrm{CK}$ & 2.320697 & 2.680524 & 2.320698 & 2.680524 \\
\hline
\end{tabular}

Table 2 Moments of the OHCEE distribution for some parameter values $\lambda_{2}=0.5$

\begin{tabular}{lllll}
\hline$\mu_{r}^{\prime}$ & $a=0.3, \lambda_{1}=0.5$ & $a=0.5, \lambda_{1}=1$ & $a=0.8, \lambda_{1}=1.2$ & $a=1, \lambda_{1}=1.8$ \\
\hline$\mu_{1}^{\prime}$ & 1.860842 & 0.943458 & 0.8113764 & 0.5553856 \\
$\mu_{2}^{\prime}$ & 4.789929 & 1.225457 & 0.8961562 & 0.4156781 \\
$\mu_{3}^{\prime}$ & 14.49713 & 1.866463 & 1.153372 & 0.360613 \\
$\mu_{4}^{\prime}$ & 48.74443 & 3.152546 & 1.639693 & 0.344453 \\
$\mu_{5}^{\prime}$ & 176.9999 & 5.743568 & 2.507746 & 0.3532041 \\
$\mu_{6}^{\prime}$ & 682.5668 & 11.10332 & 4.062111 & 0.3830325 \\
$\mathrm{SD}$ & 1.327196 & 0.335344 & 0.2378245 & 0.1072249 \\
$\mathrm{CV}$ & 0.7132234 & 0.3554414 & 0.2931124 & 0.1930639 \\
$\mathrm{CS}$ & 0.4214693 & 0.39924 & 0.3476608 & 0.303294 \\
$\mathrm{CK}$ & 2.488223 & 2.460495 & 2.404912 & 2.366611 \\
\hline
\end{tabular}

The variance, $\mathrm{CV}, \mathrm{CS}$, and $\mathrm{CK}$ are given by

$$
\begin{aligned}
& \sigma^{2}=\mu_{2}^{\prime}-\mu^{2}, \quad C V=\frac{\sigma}{\mu}=\frac{\sqrt{\mu_{2}^{\prime}-\mu^{2}}}{\mu}=\sqrt{\frac{\mu_{2}^{\prime}}{\mu^{2}}-1}, \\
& C S=\frac{E\left[(X-\mu)^{3}\right]}{\left[E(X-\mu)^{2}\right]^{3 / 2}}=\frac{\mu_{3}^{\prime}-3 \mu \mu_{2}^{\prime}+2 \mu^{3}}{\left(\mu_{2}^{\prime}-\mu^{2}\right)^{3 / 2}},
\end{aligned}
$$

and

$$
C K=\frac{E\left[(X-\mu)^{4}\right]}{\left[E(X-\mu)^{2}\right]^{2}}=\frac{\mu_{4}^{\prime}-4 \mu \mu_{3}^{\prime}+6 \mu^{2} \mu_{2}^{\prime}-3 \mu^{4}}{\left(\mu_{2}^{\prime}-\mu^{2}\right)^{2}}
$$

respectively. Table 1 lists the first six moments of the OHCEE distribution for selected values of the parameters, by fixing $\mathrm{a}=2$. And Table 2 lists the first six moments of 

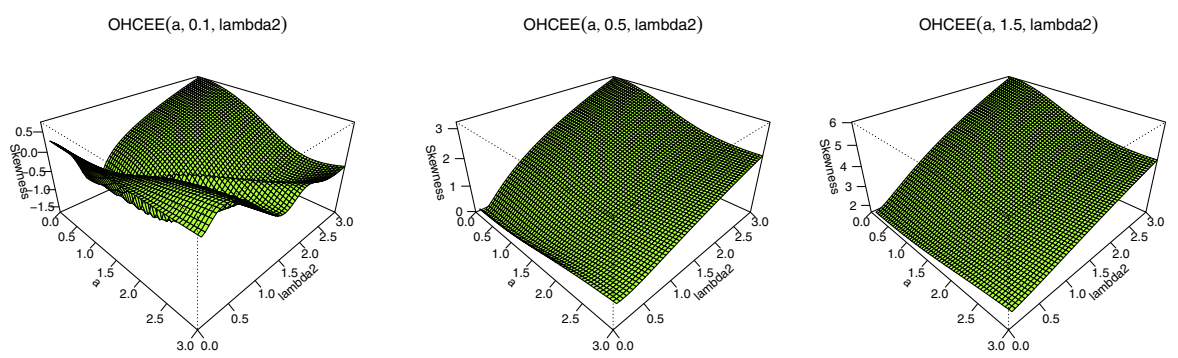

$\operatorname{OHCEE}(a, 0.1$, lambda2)

OHCEE(a, 0.5, lambda2)

$\operatorname{OHCEE}(a, 0.5$, lambda2)
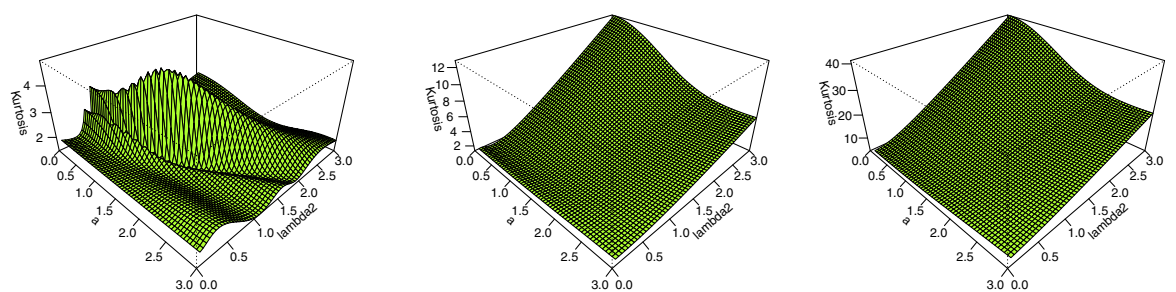

Fig. 4 3D plots of skewness and kurtosis of OHCEE distribution for some fixed values of parameter $\lambda_{1}$

the OHCEE distribution for selected values of the parameters, by fixing $\lambda_{1}=0.5$. These values can be determined numerically using R.

The moment generating function of the OHCEE distribution is given by

$E\left(e^{t X}\right)=\sum_{n=0}^{\infty} \sum_{k=0}^{2 n} \frac{2 e^{a} a^{2 n+1}\left(\begin{array}{c}2 n \\ k\end{array}\right)(-1)^{k} \lambda_{2}}{\left(e^{2 a}-1\right)(2 n) !} \frac{e^{\lambda_{2}(k+1)}}{\left(\lambda_{2}(k+1)\right)^{t / \lambda_{1}+1}} \Gamma\left(\frac{t}{\lambda_{1}}+1, \lambda_{2}(k+1)\right)$

In order to investigate and analyze amount of skewness and kurtosis of the new model under the four parameters $a, \lambda_{1}$ and $\lambda_{2}, 3 \mathrm{D}$ diagrams are presented in 3,4 and 5. Analysis of theses graphs shows that all four parameter are effective in variation of skewness and kurtosis.

\subsection{Order Statistics}

Order statistics play an important role in probability and statistics. In this section, we present the distribution of the ith order statistic from the OHCEE distribution. The pdf of the ith order statistic from the OHCEE pdf $f_{O H C E E}(x)$ is given by

$$
\begin{aligned}
f_{i: n}(x) & =\frac{n !}{(i-1) !(n-i) !} f_{\text {OHCEE }}(x)\left[F_{\text {OHCEE }}(x)\right]^{i-1}\left[1-F_{\text {OHCEE }}(x)\right]^{n-i} \\
& =\frac{n !}{(i-1) !(n-i) !} f_{O H C E E}(x) \sum_{m=0}^{n-i}\left(\begin{array}{c}
n-i \\
m
\end{array}\right)(-1)^{m}\left[F_{O H C E E}(x)\right]^{m+i-1}
\end{aligned}
$$



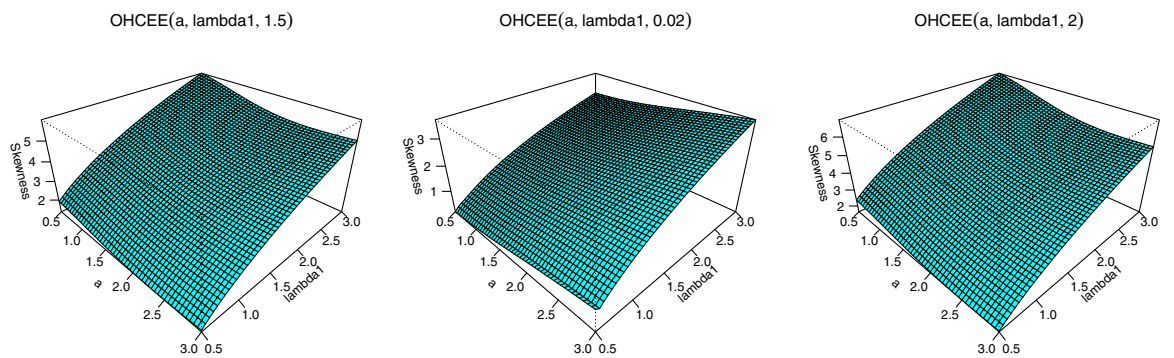

OHCEE(a, lambda1, 1.5)

$\operatorname{OHCEE}(a$, lambda1, 0.02)

$\operatorname{OHCEE}(a$, lambda1, 2)
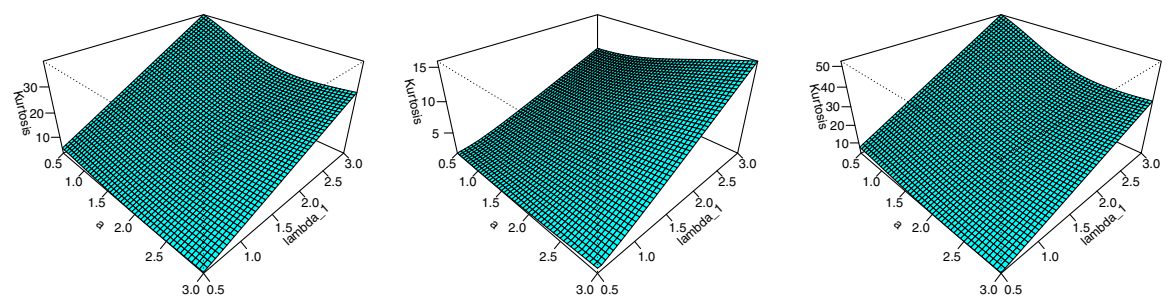

Fig. 5 3D plots of skewness and kurtosis of OHCEE distribution for some fixed values of parameter $\lambda_{2}$

Fig. 6 Scaled-TTT plot of the data set

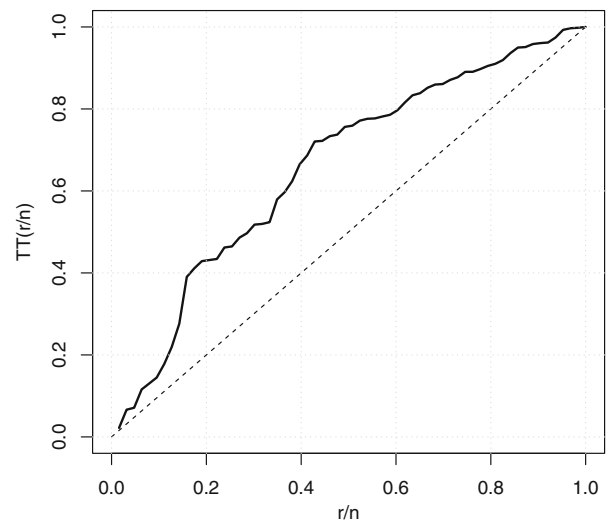

by using the binomial expansion $\left[1-F_{O H C E E}(x)\right]^{n-i}=\sum_{m=0}^{n-i}\left(\begin{array}{c}n-i \\ m\end{array}\right)(-1)^{m}$ $\left[F_{O H C E E}(x)\right]^{m}$, so that

$$
f_{i: n}(x)=\frac{1}{B(i, n-i+1)} \sum_{m=0}^{n-i}\left(\begin{array}{c}
n-i \\
m
\end{array}\right)(-1)^{m}\left[F_{O H C E E}(x)\right]^{m+i-1} f_{O H C E E}(x)
$$




\section{Infernce Procedure}

In this section, we consider estimation of unknown parameters of the $O H C E E\left(a, \lambda_{1}, \lambda_{2}\right)$ distribution by maximum likelihood method, Bayesian and bootstrap estimation. Also, we get the stress-strength parameter under this distribution.

\subsection{Maximum Likelihood Estimation}

Let $x_{1}, \ldots, x_{n}$ be a random sample from the OHCEE distribution and $\Delta=\left(a, \lambda_{1}, \lambda_{2}\right)$ the vector of parameters. The log-likelihood function is given by

$$
\begin{aligned}
L=L(\Delta)= & n \log \left(\frac{2 a e^{a}}{e^{2 a}-1}\right)+n \log \left(\lambda_{1}\right)+\lambda_{1} \sum_{i=1}^{n} x_{i}+n \log \left(\lambda_{2}\right)-\lambda_{2} \sum_{i=1}^{n}\left(e^{\lambda_{1} x_{i}}-1\right) \\
& +\sum_{i=1}^{n} \log \left(\cosh \left(a\left(1-e^{-\lambda_{2}\left(e^{\lambda_{1} x_{i}}-1\right)}\right)\right)\right)
\end{aligned}
$$

The elements of the score vector are given by

$$
\begin{aligned}
\frac{d L}{d a}= & n \frac{2 e^{3 a}(1-a)-2 e^{a}(1+a)}{2 a e^{a}\left(e^{2 a}-1\right)} \\
& +\sum_{i=1}^{n}\left(1-e^{-\lambda_{2}\left(e^{\lambda_{1} x_{i}}-1\right)}\right) \tanh \left(a\left(1-e^{-\lambda_{2}\left(e^{\lambda_{1} x_{i}}-1\right)}\right)\right)=0 \\
\frac{d L}{d \lambda_{1}}= & \frac{n}{\lambda_{1}}+\sum_{i=1}^{n} x_{i}-\lambda_{2} \sum_{i=1}^{n} x_{i} e^{\lambda_{1} x_{i}} \\
& +a \lambda_{2} \sum_{i=1}^{n} x_{i} e^{\lambda_{1} x_{i}} e^{-\lambda_{2}\left(e^{\lambda_{1} x_{i}}-1\right)} \tanh \left(a\left(1-e^{-\lambda_{2}\left(e^{\lambda_{1} x_{i}}-1\right)}\right)\right)=0
\end{aligned}
$$

and

$$
\begin{aligned}
\frac{d L}{d \lambda_{2}}= & \frac{n}{\lambda_{2}}-\sum_{i=1}^{n}\left(e^{\lambda_{1} x_{i}}-1\right) \\
& +a \sum_{i=1}^{n}\left(e^{\lambda_{1} x_{i}}-1\right) e^{-\lambda_{2}\left(e^{\lambda_{1} x_{i}}-1\right)} \tanh \left(a\left(1-e^{-\lambda_{2}\left(e^{\lambda_{1} x_{i}}-1\right)}\right)\right)=0
\end{aligned}
$$

respectively.

The maximum likelihood estimates, $\hat{\Delta}$ of $\Delta=\left(a, \lambda_{1}, \lambda_{2}\right)$ are obtained by solving the nonlinear equations $\frac{d L}{d a}=0, \frac{d L}{d \lambda_{1}}=0, \frac{d L}{d \lambda_{2}}=0$. hese equations are not in closed form and the values of the parameters a, $\lambda_{1}$ and $\lambda_{2}$ must be found by using iterative methods. Therefore, the maximum likelihood estimates, $\hat{\Delta}$ of $\Delta=\left(a, \lambda_{1}, \lambda_{2}\right)$ can be determined using an iterative method such as the NewtonRaphson procedure. 


\subsection{Stress-Strength Parameter Estimation}

In reliability, the stress-strength model describes the life of a component which has a random strength $\mathrm{X}$ subjected to a random stress $\mathrm{Y}$. In this section, we consider the problem of estimating $R=P(X>Y)$, under the assumption that $X \sim \operatorname{OHCEE}\left(a_{1}, \lambda_{11}, \lambda_{21}\right), Y \sim \operatorname{OHCEE}\left(a_{2}, \lambda_{12}, \lambda_{22}\right)$, and $\mathrm{X}$ and $\mathrm{Y}$ are independently distributed. Then, we can get

$$
\begin{aligned}
R=P(X>Y)= & \int_{0}^{\infty}\left[1-H_{X}(y)\right] h_{Y}(y) \mathrm{d} y \\
= & \int_{0}^{\infty}\left[1-\frac{2 e_{1}^{a}}{e^{2 a_{1}}-1} \sinh \left(a_{1}\left(1-e^{-\lambda_{21}\left(e^{\lambda_{11} y}-1\right)}\right)\right)\right] \\
& \times \frac{2 a_{2} e^{a_{2}}}{e^{2 a_{2}}-1} \lambda_{12} e^{\lambda_{12} y} \lambda_{22} e^{-\lambda_{22}\left(e^{\lambda_{12} y}-1\right)} \cosh \left(a_{2}\left(1-e^{-\lambda_{22}\left(e^{\lambda_{12} y}-1\right)}\right)\right) \mathrm{d} y
\end{aligned}
$$

To compute the MLE of R, let us first obtain the MLEs of $a_{1}, a_{2}, \lambda_{11}, \lambda_{12}, \lambda_{21}$ and $\lambda_{22}$. Suppose $x_{1}, \ldots, x_{n}$ be the observed values of a random sample of size $\mathrm{n}$ from $O H C E E\left(a_{1}, \lambda_{11}, \lambda_{12}\right)$ and $y_{1}, \ldots, y_{m}$ be the observed values of a random sample of size $\mathrm{m}$ from $O H C E E\left(a_{2}, \lambda_{12}, \lambda_{22}\right)$. Therefore, the log-likelihood function of $a_{1}, a_{2}, \lambda_{11}, \lambda_{12}, \lambda_{21}$ and $\lambda_{22}$ is given by

$$
\begin{aligned}
L\left(a_{1}, a_{2}, \lambda_{11}, \lambda_{12}, \lambda_{21}, \lambda_{22}\right)= & n \log \left(\frac{2 a_{1} e_{1}^{a}}{e^{2 a_{1}}-1}\right) \\
& +n \log \left(\lambda_{11}\right)+\lambda_{11} \sum_{i=1}^{n} x_{i}+n \log \left(\lambda_{21}\right) \\
& -\lambda_{21} \sum_{i=1}^{n}\left(e^{\lambda_{11} x_{i}}-1\right) \\
& +\sum_{i=1}^{n} \log \left(\cosh \left(a_{1}\left(1-e^{-\lambda_{21}\left(e^{\lambda_{11} x_{i}}-1\right)}\right)\right)\right) \\
& +m \log \left(\frac{2 a_{2} e_{2}^{a}}{e^{2 a_{2}}-1}\right) \\
& +m \log \left(\lambda_{12}\right)+\lambda_{12} \sum_{i=1}^{m} y_{i}+m \log \left(\lambda_{22}\right) \\
& -\lambda_{22} \sum_{i=1}^{m}\left(e^{\lambda_{12} y_{i}}-1\right) \\
& +\sum_{i=1}^{m} \log \left(\cosh \left(a_{2}\left(1-e^{-\lambda_{22}\left(e^{\lambda_{12} y_{i}}-1\right)}\right)\right)\right)
\end{aligned}
$$

It follows that the MLEs of $a_{1}, a_{2}, \lambda_{11}, \lambda_{12}, \lambda_{21}$ and $\lambda_{22}$, say $\hat{a}_{1}, \hat{a}_{2}, \hat{\lambda}_{11}, \hat{\lambda}_{12}, \hat{\lambda}_{21}$ and $\hat{\lambda}_{22}$, are the simultaneous solutions of the following equations: 


$$
\begin{aligned}
& n \frac{2 e^{3 a_{1}}\left(1-a_{1}\right)-2 e_{1}^{a}\left(1+a_{1}\right)}{2 a_{1} e_{1}^{a}\left(e^{2 a_{1}}-1\right)}+\sum_{i=1}^{n}\left(1-e^{-\lambda_{12}\left(e^{\lambda_{11} x_{i}}-1\right)}\right) \tanh \left(a_{1}\left(1-e^{-\lambda_{12}\left(e^{\lambda_{11} x_{i}}-1\right)}\right)\right)=0, \\
& m \frac{2 e^{3 a_{2}}\left(1-a_{2}\right)-2 e_{2}^{a}\left(1+a_{2}\right)}{2 a_{2} e_{2}^{a}\left(e^{2 a_{2}}-1\right)}+\sum_{i=1}^{m}\left(1-e^{-\lambda_{22}\left(e^{\lambda_{12} y_{i}}-1\right)}\right) \tanh \left(a_{2}\left(1-e^{-\lambda_{22}\left(e^{\lambda_{12} y_{i}}-1\right)}\right)\right)=0, \\
& \frac{n}{\lambda_{11}}+\sum_{i=1}^{n} x_{i}-\lambda_{21} \sum_{i=1}^{n} x_{i} e^{\lambda_{11} x_{i}}+a_{1} \lambda_{21} \sum_{i=1}^{n} x_{i} e^{\lambda_{11} x_{i}} e^{-\lambda_{21}\left(e^{\lambda_{1} x_{i}}-1\right)} \tanh \left(a_{1}\left(1-e^{-\lambda_{21}\left(e^{\lambda_{1} x_{i}}-1\right)}\right)\right)=0, \\
& \frac{m}{\lambda_{12}}+\sum_{i=1}^{m} y_{i}-\lambda_{22} \sum_{i=1}^{m} y_{i} e^{\lambda_{12} y_{i}}+a_{2} \lambda_{22} \sum_{i=1}^{m} y_{i} e^{\lambda_{12} y_{i}} e^{-\lambda_{22}\left(e^{\lambda_{1} y_{i}}-1\right)} \tanh \left(a_{2}\left(1-e^{-\lambda_{22}\left(e^{\lambda_{12} y_{i}}-1\right)}\right)\right)=0, \\
& \frac{n}{\lambda_{21}}-\sum_{i=1}^{n}\left(e^{\lambda_{11} x_{i}}-1\right)+a_{1} \sum_{i=1}^{n}\left(e^{\lambda_{11} x_{i}}-1\right) e^{-\lambda_{21}\left(e^{\lambda_{11} x_{i}}-1\right)} \tanh \left(a_{1}\left(1-e^{-\lambda_{21}\left(e^{\lambda_{11} x_{i}}-1\right)}\right)\right)=0 \\
& \frac{m}{\lambda_{22}}-\sum_{i=1}^{m}\left(e^{\lambda_{12} y_{i}}-1\right)+a_{2} \sum_{i=1}^{m}\left(e^{\lambda_{12} y_{i}}-1\right) e^{-\lambda_{22}\left(e^{\lambda_{12} y_{i}}-1\right)} \tanh \left(a_{2}\left(1-e^{-\lambda_{22}\left(e^{\lambda_{12} y_{i}}-1\right)}\right)\right)=0
\end{aligned}
$$

Once we obtain $\hat{a}_{1}, \hat{a}_{2}, \hat{\lambda}_{11}, \hat{\lambda}_{12}, \hat{\lambda}_{21}$ and $\hat{\lambda}_{22}$ therefore, we compute the MLE of R as $\hat{R}$.

Here, the maximum likelihood approach does not give an explicit estimator for the MLEs of parameters and hence the MLE of R. In practice, one has to use numerical methods to find the MLEs, such methods are well implemented in R software packages.

\subsection{Bootstrap Estimation}

The parameters of the fitted distribution can be estimated by parametric (resampling from the fitted distribution) or non-parametric (resampling with replacement from the original data set) bootstraps resampling (see [5]). These two parametric and nonparametric bootstrap procedures are described as follows.

\section{Parametric bootstrap procedure:}

1. Estimate $\theta$ (vector of unknown parameters), say $\hat{\theta}$, by using the $M L E$ procedure based on a random sample.

2. Generate a bootstrap sample $\left\{X_{1}^{*}, \ldots, X_{m}^{*}\right\}$ using $\hat{\theta}$ and obtain the bootstrap estimate of $\theta$, say $\widehat{\theta^{*}}$, from the bootstrap sample based on the $M L E$ procedure.

3. Repeat Step 2 NBOOT times.

4. Order $\widehat{\theta}^{*}{ }_{1}, \ldots, \widehat{\theta}^{*}{ }_{N B}$ BOOT as $\widehat{\theta}^{*}{ }_{(1)}, \ldots, \widehat{\theta}^{*}{ }_{(N B O O O T)}$. Then obtain $\gamma$-quantiles and $100(1-\alpha) \%$ confidence intervals of parameters.

In case of the $O H C E E$ distribution, the parametric bootstrap estimators (PBs) of $\alpha, \beta, \lambda$ and $\xi$, say $\hat{\alpha}_{P B}, \hat{\beta}_{P B}, \hat{\lambda}_{P B}$ and $\hat{\xi}_{P B}$, respectively.

\section{Nonparametric bootstrap procedure}

1. Generate a bootstrap sample $\left\{X_{1}^{*}, \ldots, X_{m}^{*}\right\}$, with replacement from original data set.

2. Obtain the bootstrap estimate of $\theta$ with MLE procedure, say $\widehat{\theta^{*}}$, by using the bootstrap sample.

3. Repeat Step 2 NBOOT times. 
4. Order $\widehat{\theta}^{*}{ }_{1}, \ldots, \widehat{\theta}^{*}{ }_{N B O O T}$ as $\widehat{\theta}^{*}{ }_{(1)}, \ldots, \widehat{\theta}^{*}{ }_{(N B O O T)}$. Then obtain $\gamma$-quantiles and $100(1-\alpha) \%$ confidence intervals of parameters.

In case of the $O H C E E$ distribution, the nonparametric bootstrap estimators (NPBs) of $\alpha, \beta, \lambda$ and $\xi$, say $\hat{\alpha}_{N P B}, \hat{\beta}_{N P B}, \hat{\lambda}_{N P B}$ and $\hat{\xi}_{N P B}$, respectively.

\subsection{Bayesian Estimation}

In this section we consider Bayesian inference of the unknown parameters of the $O H C E E\left(a, \lambda_{1}, \lambda_{2}\right)$. It is assumed that a is known and both of the parameters of $\lambda_{1}$ and $\lambda_{2}$ are unknown and have the independent gamma prior distributions with probability density functions

$$
g\left(\lambda_{1}\right) \propto e^{-c \lambda_{1}} \lambda_{1}^{b-1}, \quad \lambda_{1}>0, \quad b, c>0,
$$

and

$$
g\left(\lambda_{2}\right) \propto e^{-s \lambda_{2}} \lambda_{2}^{d-1}, \quad \lambda_{2}>0, \quad s, d>0,
$$

Thus, the joint prior distribution for $\lambda_{1}$ and $\lambda_{2}$ is

$$
g\left(\lambda_{1}, \lambda_{2}\right) \propto \lambda_{1}^{b-1} \lambda_{2}^{d-1} e^{-\left(c \lambda_{1}+s \lambda_{2}\right)}, \quad \lambda_{1}>0, \quad \lambda_{2}>0, \quad b, c, s, d>0
$$

Now, the posterior density function of $\lambda_{1}$ and $\lambda_{2}$ given the data, denoted by $\pi\left(\lambda_{1}, \lambda_{2} \mid \underline{x}\right)$, can be obtained the following as

$$
\begin{aligned}
\pi\left(\lambda_{1}, \lambda_{2} \mid \underline{x}\right)= & \frac{1}{R(\underline{x})} \lambda_{1}^{n+b-1} \lambda_{2}^{n+d-1} e^{-c \lambda_{1}} e^{-\left(s+\sum_{i=1}^{n}\left(e^{\lambda_{1} x_{i}}-1\right)\right)} e^{\lambda_{1} \sum_{i=1}^{n} x_{i}} \\
& \times \prod_{i=1}^{n} \cosh \left(a\left(1-e^{-\lambda_{2}\left(e^{\lambda_{1} x_{i}}-1\right)}\right)\right), \quad \lambda_{1}>0, \lambda_{2}>0
\end{aligned}
$$

where

$$
\begin{aligned}
R(\underline{x})= & \int_{0}^{\infty} \int_{0}^{\infty} \lambda_{1}^{n+b-1} \lambda_{2}^{n+d-1} e^{-c \lambda_{1}} e^{-\left(s+\sum_{i=1}^{n}\left(e^{\lambda_{1} x_{i}}-1\right)\right)} e^{\lambda_{1} \sum_{i=1}^{n} x_{i}} \\
& \times \prod_{i=1}^{n} \cosh \left(a\left(1-e^{-\lambda_{2}\left(e^{\lambda_{1} x_{i}}-1\right)}\right)\right) \mathrm{d} \lambda_{1} \mathrm{~d} \lambda_{2}
\end{aligned}
$$

Therefore, the Bayes estimate of any function of $\alpha$ and $\beta$, say $\theta(\alpha, \beta)$ under squared error loss function is 
Table 3 Bayes estimator and posterior risk under different loss functions

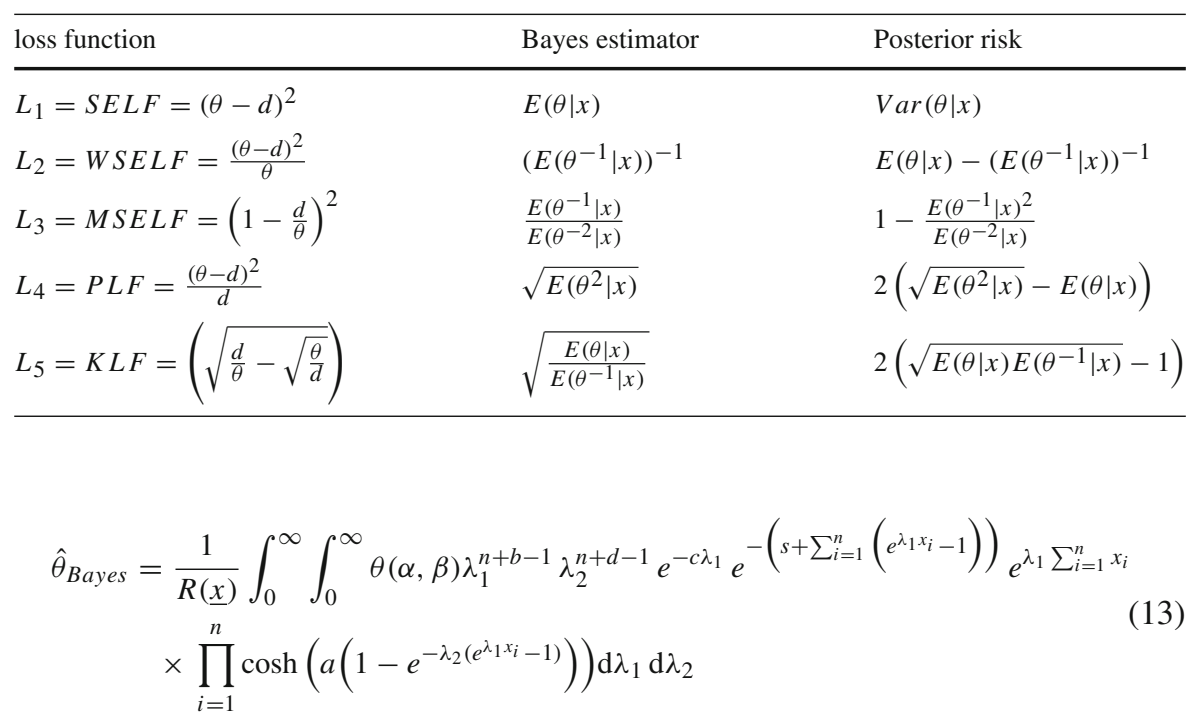

\subsubsection{Lindleys Approximation}

It is impossible to compute Eq. (2) analytically. Lindleys approximation is used to compute the ratio of integrals of the form (1). Based on Lindleys approximation, the approximate Bayes estimates of $\lambda_{1}$ and $\lambda_{2}$ under squared error loss function are

$$
\begin{aligned}
\hat{\lambda_{1 \text { Lindley }}=} & \hat{\lambda_{1}}+\frac{1}{2}\left[2 \rho_{\lambda_{1}} \sigma_{\lambda_{1} \lambda_{1}}+2 \rho_{\lambda_{2}} \sigma_{\lambda_{1} \lambda_{2}}+\sigma_{\lambda_{1} \lambda_{1}}\left(L_{\lambda_{1} \lambda_{1} \lambda_{1}} \sigma_{\lambda_{1} \lambda_{1}}\right.\right. \\
& \left.+L_{\lambda_{1} \lambda_{2} \lambda_{1}} \sigma_{\lambda_{1} \lambda_{2}}+L_{\lambda_{2} \lambda_{1} \lambda_{1}} \sigma_{\lambda_{2} \lambda_{1}}+L_{\lambda_{2} \lambda_{2} \lambda_{1}} \sigma_{\lambda_{2} \lambda_{1}}\right) \\
& +\sigma_{\lambda_{2} \lambda_{1}}\left(L_{\lambda_{1} \lambda_{1} \lambda_{2}} \sigma_{\lambda_{1} \lambda_{1}}+L_{\lambda_{1} \lambda_{1} \lambda_{2}} \sigma_{\lambda_{1} \lambda_{2}}+L_{\lambda_{2} \lambda_{1} \lambda_{2}} \sigma_{\lambda_{2} \lambda_{1}}+L_{\lambda_{2} \lambda_{2} \lambda_{2}} \sigma_{\lambda_{2} \lambda_{2}}\right]
\end{aligned}
$$

and

$$
\begin{aligned}
\hat{\lambda_{2} \text { Lindley }}= & \hat{\lambda_{2}}+\frac{1}{2}\left[2 \rho_{\lambda_{1}} \sigma_{\lambda_{2} \lambda_{1}}+2 \rho_{\lambda_{2}} \sigma_{\lambda_{2} \lambda_{2}}+\sigma_{\lambda_{1} \lambda_{2}}\left(L_{\lambda_{1} \lambda_{1} \lambda_{1}} \sigma_{\lambda_{1} \lambda_{1}}\right.\right. \\
& \left.+L_{\lambda_{1} \lambda_{2} \lambda_{1}} \sigma_{\lambda_{1} \lambda_{2}}+L_{\lambda_{2} \lambda_{1} \lambda_{1}} \sigma_{\lambda_{2} \lambda_{1}}+L_{\lambda_{2} \lambda_{2} \lambda_{1}} \sigma_{\lambda_{2} \lambda_{2}}\right) \\
& +\sigma_{\lambda_{2} \lambda_{2}}\left(L_{\lambda_{1} \lambda_{1} \lambda_{2}} \sigma_{\lambda_{1} \lambda_{1}}+L_{\lambda_{1} \lambda_{2} \lambda_{2}} \sigma_{\lambda_{1} \lambda_{2}}+L_{\lambda_{2} \lambda_{1} \lambda_{2}} \sigma_{\lambda_{2} \lambda_{1}}+L_{\lambda_{2} \lambda_{2} \lambda_{2}} \sigma_{\lambda_{2} \lambda_{2}}\right]
\end{aligned}
$$

respectively. Here $\hat{\lambda}_{1}$ and $\hat{\lambda_{2}}$ are the MLEs of $\lambda_{1}$ and $\lambda_{2}$, respectively. The details involved in the derivations of Eqs. (3) and (4) are placed in the "Appendix" by a function of the parameter and corresponding estimator. Five well-known loss functions and associated Bayesian estimators and corresponding posterior risk are presented in Table 3 . 


\section{Algorithm and a Simulation Study}

In this section, we give one algorithm for generating the random data $x_{1}, \ldots, x_{n}$ from the OHCEE distribution and hence a simulation study is obtained to evaluate the performance of MLEs.

\subsection{Algorithm}

Here, we obtain a algorithm for generating the random data $x_{1}, \ldots, x_{n}$ from the OHCEE distribution as follows. The algorithm is based on generating random data from the inverse cdf of the OHCEE distribution.

\section{Algorithm}

1. Generate $U_{i} \sim$ Uniform $(0,1) ; i=1, \ldots, n$,

2. set

$$
X_{p}=\frac{1}{\lambda_{1}} \log \left(1-\frac{1}{\lambda_{2}} \log \left(1-\frac{1}{a} \log \left(\frac{e^{2 a}-1}{2 e^{a}} p+\sqrt{\left(\frac{e^{2 a}-1}{2 e^{a}} p\right)^{2}+1}\right)\right) ; 0 \leq p \leq 1\right.
$$

\subsection{Monte Carlo Simulation Study}

In this section, we assess the performance of the MLE's of the parameters with respect to sample size $\mathrm{n}$ for the $O H C E E\left(a, \lambda_{1}, \lambda_{2}\right)$ distribution. We used above Algorithm to generate data from the OHCEE distribution. The assessment of performance is based on a simulation study by using the Monte Carlo method. Let $\hat{a}, \hat{\lambda_{1}}$ and $\hat{\lambda_{2}}$ be the MLEs of the parameters a, $\lambda_{1}$ and $\lambda_{2}$, respectively. We compute the mean square error (MSE) and bias of the MLEs of the parameters a, $\lambda_{1}$ and $\lambda_{2}$, based on the simulation results of $\mathrm{N}=2000$ independence replications. results are summarized in Table 4 for different values of $n, a, \lambda_{1}$ and $\lambda_{2}$. From Table 4 the results verify that MSE and bias of the MLEs of the parameters decrease with respect to sample size $\mathrm{n}$ increases. Hence, we can see the MLEs of $\mathrm{a}, \lambda_{1}$ and $\lambda_{2}$, are consistent estimators.

\section{Practical Data Applications}

In this section, we present the application of the OHCEE model to one practical data set to illustrate its flexibility among a set of competitive models (Table 6).

The windshield on a large aircraft is a complex piece of equipment, comprised basically of several layers of material, including a very strong outer skin with a heated layer just beneath it, all laminated under high temperature and pressure. Failures of these items are not structural failures. Instead, they typically involve damage or delamination of the nonstructural outer ply or failure of the heating system. These failures do not result in damage to the aircraft but do result in replacement of the 
Table 4 MSEs and Average biases (values in parentheses) of the simulated estimates

\begin{tabular}{|c|c|c|c|c|}
\hline & & $\mathrm{a}=0.3$ & $\lambda_{1}=0.5$ & $\lambda_{2}=1.5$ \\
\hline \multirow[t]{5}{*}{$\mathrm{n}$} & 30 & $0.8189(0.1738)$ & $0.0865(0.0544)$ & $132.3321(2.3249)$ \\
\hline & 50 & $0.7216(0.1095)$ & $0.0519(0.0187)$ & $40.7226(1.3271)$ \\
\hline & 100 & $0.6768(0.1396)$ & $0.0275(-0.0182)$ & $15.1627(0.9064)$ \\
\hline & 200 & $0.5902(0.1135)$ & $0.0172(-0.0303)$ & $4.4592(0.6561)$ \\
\hline & & $\mathrm{a}=0.5$ & $\lambda_{1}=1$ & $\lambda_{2}=2$ \\
\hline \multirow[t]{5}{*}{$\mathrm{n}$} & 30 & $1.0717(0.1182)$ & $0.4595(0.1103)$ & $353.7897(4.8048)$ \\
\hline & 50 & $0.8683(0.0302)$ & $0.2869(0.0377)$ & 165.9069 \\
\hline & 100 & $0.7678(0.0574)$ & $0.1643(-0.0512)$ & $65.0995(2.2592)$ \\
\hline & 200 & $0.6099(0.0405)$ & $0.1014(-0.0595)$ & $17.2459(1.2489)$ \\
\hline & & $a=0.8$ & $\lambda_{1}=0.5$ & $\lambda_{2}=1.5$ \\
\hline \multirow[t]{5}{*}{$\mathrm{n}$} & 30 & $1.2207(-0.0169)$ & $0.0873(0.0515)$ & $106.8476(2.3648)$ \\
\hline & 50 & $1.0508(-0.0300)$ & $0.0538(0.0134)$ & $54.7730(1.7901)$ \\
\hline & 100 & $0.8062(-0.0321)$ & $0.0304(-0.0151)$ & $17.3773(1.1130)$ \\
\hline & 200 & $0.6423(-0.0515)$ & $0.0196(-0.0180)$ & $4.9051(0.6426)$ \\
\hline & & $a=1$ & $\lambda_{1}=1.5$ & $\lambda_{2}=2$ \\
\hline \multirow[t]{5}{*}{$\mathrm{n}$} & 30 & $1.4442(-0.0288)$ & $1.0211(0.1839)$ & $297.3460(4.7064)$ \\
\hline & 50 & $1.1301(-0.0819)$ & $0.7082(0.0773)$ & $163.7686(3.5522)$ \\
\hline & 100 & $0.8579(-0.0866)$ & $0.4011(-0.0087)$ & $64.1250(2.0630)$ \\
\hline & 200 & $0.6941(-0.1346)$ & $0.2789(-0.0210)$ & $26.7817(1.2582)$ \\
\hline & & $\mathrm{a}=2$ & $\lambda_{1}=2$ & $\lambda_{2}=1.5$ \\
\hline \multirow[t]{4}{*}{$\mathrm{n}$} & 30 & $2.3294(-0.1836)$ & $2.1399(0.5090)$ & $223.8817(3.3442)$ \\
\hline & 50 & $1.6476(-0.2089)$ & $1.4322(0.3452)$ & $57.4308(1.7158)$ \\
\hline & 100 & $1.1889(-0.2213)$ & $0.8673(0.2137)$ & $12.2516(0.6404)$ \\
\hline & 200 & $0.9679(-0.2788)$ & $0.6181(0.2014)$ & $3.7672(0.2618)$ \\
\hline
\end{tabular}

windshield. We consider the data on service times for a particular model windshield given in Table 16.11 of Murthy et al. [8]. These data were recently studied by Ramos et al. [9]. These data are:

0.0461 .4362 .5920 .1401 .4922 .6000 .1501 .5802 .6700 .2481 .7192 .7170 .2801 .794 2.8190 .3131 .9152 .8200 .3891 .9202 .8780 .4871 .9632 .9500 .6221 .9783 .0030 .900 2.0533 .1020 .9522 .0653 .3040 .9962 .1173 .4831 .0032 .1373 .5001 .0102 .1413 .622 1.0852 .1633 .6651 .0922 .1833 .6951 .1522 .2404 .0151 .1832 .3414 .6281 .2442 .435 4.8061 .2492 .4644 .8811 .2622 .5435 .140 .

Graphical measure The total time test (TTT) plot due to Aarset [3] is an important graphical approach to verify whether the data can be applied to a specific distribution or not. According to Aarset [3], the empirical version of the TTT plot is given by plotting $T(r / n)=\left[\sum_{i=1}^{r} y_{i: n}+(n-r) y_{r: n}\right] / \sum_{i=1}^{n} y_{i: n}$ against $r / n$, where $r=1, \ldots, n$ 
Table 5 Bootstrap point and interval estimation of the parameters a, $\lambda_{1}$ and $\lambda_{2}$

\begin{tabular}{llllll}
\hline & Parametric bootstrap & & & \multicolumn{2}{l}{ Non-parametric bootstrap } \\
\cline { 2 - 3 } & Point estimation & CI & & Point estimation & CI \\
\hline a & 2.735 & $(0.464,4.430)$ & & 2.641 & $(0.672,4.063)$ \\
$\lambda_{1}$ & 0.501 & $(0.248,0.967)$ & & 0.497 & $(0.324,0.847)$ \\
$\lambda_{2}$ & 0.910 & $(0.136,3.224)$ & & 0.927 & $(0.188,2.080)$ \\
\hline
\end{tabular}

and $y_{i: n}(i=1, \ldots, n)$ are the order statistics of the sample. Aarset [3] showed that the hazard function is constant if the TTT plot is graphically presented as a straight diagonal, the hazard function is increasing (or decreasing) if the TTT plot is concave (or convex). The hazard function is U-shaped if the TTT plot is convex and then concave, if not, the hazard function is unimodal. The TTT plots for data set is presented in Fig. 6. These plots indicate that the empirical hazard rate functions of the data set is increasing. Therefore, the OHCEE distribution is appropriate to fit this data set. parametric and non-parametric bootstrap methods for the real data set. We provide results of bootstrap estimation based on 10,000 bootstrap replicates in Table 5 .

We fit the OHCEE distribution to the one data set and compare it with the HCE, gamma, generalized exponential and Weibull densities. Table 6 shows the MLEs of parameters, log-likelihood, Akaike information criterion (AIC), Cramrvon $\operatorname{Mises}\left(W^{*}\right)$, AndersonDarling $\left(A^{*}\right)$ and $\mathrm{p}$-value $(\mathrm{P})$ statistics for the data set. The OHCEE distribution provides the best fit for the data set as it shows the lowest $\mathrm{AIC}, A^{*}$ and $W^{*}$ than other considered models. The relative histograms, fitted OHCEE, HCE, gamma, generalized exponential and Weibull PDFs for data are plotted in Fig. 7. The plots of empirical and fitted survival functions, P-P plots and Q-Q plots for the OHCEE and other fitted distributions are displayed in Figs. 7 and 8 respectively. These plots also support the results in Table 6. We compare the OHCEE model with a set of competitive models, namely:

(i) Hyperbolic Cosine-Exponential distribution (HCE) [6]. The two-parameter HCE density function is given by

$$
f(x ; a, \lambda)=\frac{2 a e^{a}}{e^{2 a}-1} \lambda e^{-\lambda x} \cosh \left(a\left(1-e^{-\lambda x}\right)\right) ; \quad x>0
$$

where $a>0$ and $\lambda>0$.

(ii) The two-parameter Weibull distribution is given by

$$
f(x ; \alpha, \beta)=\frac{\alpha}{\beta}\left(\frac{x}{\beta}\right)^{\alpha-1} e^{-\left(\frac{x}{\beta}\right)^{\alpha}} ; x>0
$$

where $\alpha>0$ and $\beta>0$.

(iii) The two-parameter Gamma distribution is given by

$$
f(x ; \alpha, \theta)=\frac{1}{\theta^{\alpha} \Gamma(\alpha)} x^{\alpha-1} e^{-(x / \theta)} ; \quad x>0
$$


Histogram and theoretical densities

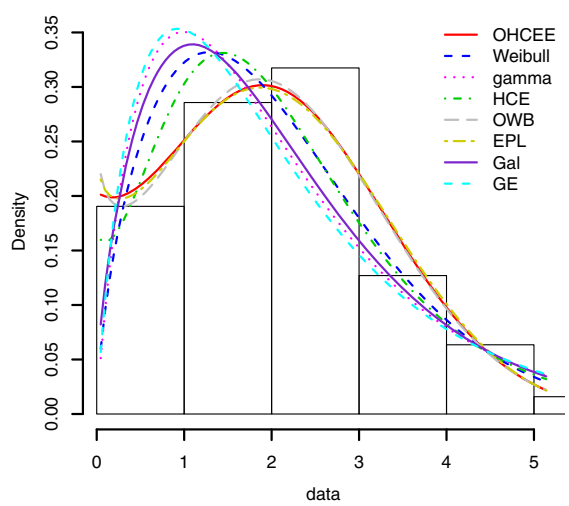

Empirical and theoretical CDFs

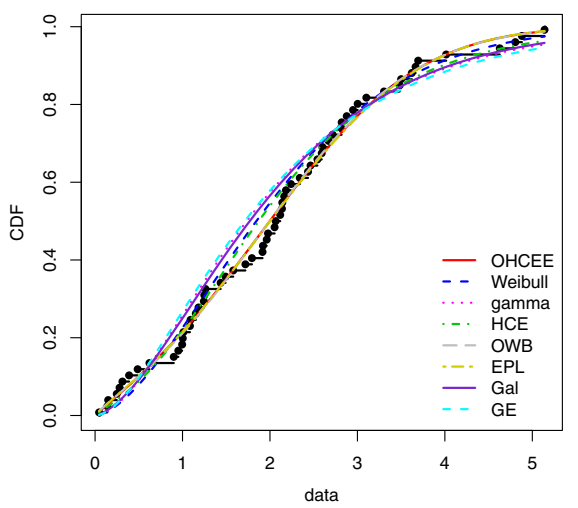

Fig. 7 Estimated densities and Empirical and Estimated cdf for the data set
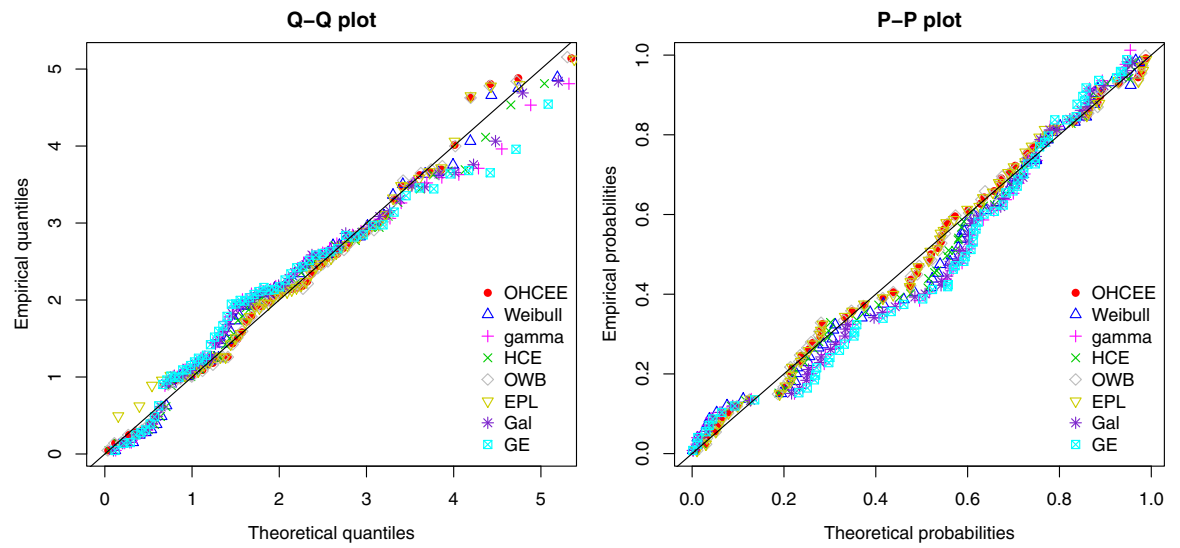

Fig. 8 Q-Q and P-P plots for the data set

where $\alpha>0$ and $\theta>0$ and $\Gamma(\alpha)=\int_{0}^{\infty} t^{\alpha-1} e^{-t} \mathrm{~d} t$.

(iv) The two-parameter generalized exponential (GE) distribution is given by

$$
f(x ; \alpha, \lambda)=\alpha \lambda e^{-\lambda x}\left(1-e^{-\lambda x}\right)^{\alpha-1} ; \quad x>0
$$

where $\alpha>0$ and $\lambda>0$.

As mentioned in inference section, there are not closed expression for MLE estimation of parameters $a, \lambda_{1}$ and $\lambda_{2}$. We use numerical methods to obtain MLE estimation of these parameters. To evaluate the results of MLE estimation (Table 6), We provide profile-likelihood plots of $O H C E E$ distribution for each parameter in Fig. 9.

The corresponding Bayesian point estimation and posterior risk provided in Table 7. 
Table 6 Parameter estimates (standard errors),log-likelihood values and goodness of fit measures

\begin{tabular}{lllllllll}
\hline Model & MLEs of parameters (s.e) & Log-likelihood & AIC & BIC & $A^{*}$ & $W^{*}$ & K.S & P \\
\hline OHCEE & $\hat{a}=2.58(0.96)$ & -97.91 & 201.83 & 208.26 & 0.30 & 0.04 & 0.06 & 0.95 \\
& $\hat{\lambda}_{1}=0.24(0.14)$ & & & & & & & \\
& $\hat{\lambda}_{2}=2.08(2.02)$ & & & & & & & \\
HCE & $\hat{a}=3.69(0.67)$ & -99.81 & 203.63 & 207.92 & 0.45 & 0.07 & 0.10 & 0.51 \\
& $\hat{\lambda}=0.89(0.09)$ & & & & & & & \\
Weibull & $\hat{\alpha}=1.62(0.16)$ & -100.31 & 204.63 & 208.92 & 0.64 & 0.09 & 0.10 & 0.41 \\
& $\hat{\beta}=2.30(0.18)$ & & & & & & & \\
Gamma & $\hat{\alpha}=1.9(0.31)$ & -102.83 & 209.66 & 213.95 & 1.16 & 0.2 & 0.58 & 0.84 \\
& $\hat{\theta}=0.91(0.17)$ & & & & & & & \\
GE & $\hat{\alpha}=1.89(0.34)$ & -103.54 & 211.09 & 215.37 & 1.31 & 0.23 & 0.14 & 0.13 \\
& $\hat{\lambda}=0.69(0.09)$ & & & & & & & \\
\hline
\end{tabular}
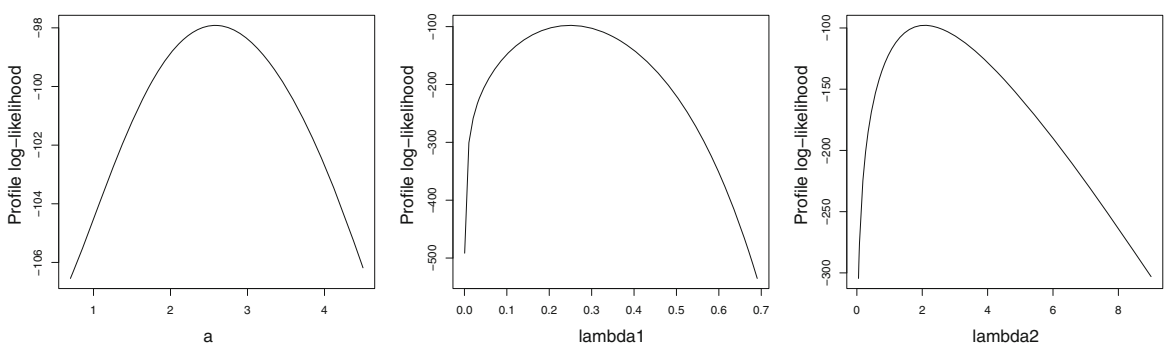

Fig. 9 profile-likelihood plots of OHCEE distribution

Table 7 Bayesian estimates and their posterior risks of parameters under different loss functions based on real data

\begin{tabular}{|c|c|c|c|c|}
\hline \multirow{3}{*}{$\begin{array}{l}\text { Data } \\
\text { Bayes } \\
\text { Loss functions }\end{array}$} & \multicolumn{4}{|c|}{ Service times } \\
\hline & \multicolumn{2}{|l|}{$\widehat{\widehat{\lambda_{1}}}$} & \multicolumn{2}{|l|}{$\widehat{\lambda_{2}}$} \\
\hline & Estimate & Risk & Estimate & Risk \\
\hline SELF & 0.242722 & 0.00022 & 2.140396 & 0.04963 \\
\hline WSELF & 0.243575 & 0.00085 & 2.165938 & 0.02554 \\
\hline MSELF & 0.244380 & 0.00330 & 2.195038 & 0.01343 \\
\hline PLF & 0.242265 & 0.00091 & 2.128769 & 0.02325 \\
\hline KLF & 0.243148 & 0.00350 & 2.153129 & 0.01182 \\
\hline
\end{tabular}

\section{Conclusion}

In this article, a new model for the lifetime distributions is introduced and its main properties are discussed. A special submodel of this family is taken up by considering exponential distributions in place of the parent distribution $\mathrm{F}$ and instead of the parent distribution G. We also show that the proposed distribution has variability of hazard 
rate shapes such as increasing and upside-down bathtub shapes. Numerical results of maximum likelihood, Bayesian and bootstrap procedures for a set of real data are presented in separate tables. From a practical point of view, we show that the proposed distribution is more flexible than some common statistical distributions.

Open Access This article is distributed under the terms of the Creative Commons Attribution 4.0 International License (http://creativecommons.org/licenses/by/4.0/), which permits unrestricted use, distribution, and reproduction in any medium, provided you give appropriate credit to the original author(s) and the source, provide a link to the Creative Commons license, and indicate if changes were made.

\section{Appendix}

Lindley developed an asymptotic expansion for evaluating the ratio of integrals of the form

$$
u^{*}=E[u(\alpha, \beta) \mid x]=\frac{\iint u(\alpha, \beta) \exp [l(\alpha, \beta \mid x)+\rho(\alpha, \beta)]}{\iint \exp [l(\alpha, \beta \mid x)+\rho(\alpha, \beta)]} \mathrm{d} \alpha \mathrm{d} \beta,
$$

where $u(\alpha, \beta)$ is a function of $\alpha$ and $\beta$ only, and $l(\alpha, \beta \mid x)$ is the log-likelihood function and $\rho(\alpha, \beta)=\log [\pi(\alpha, \beta)]$.

Utilizing Lindleys method, $u^{*}$ can be approximated as

$$
\begin{aligned}
u^{*}=u+\frac{1}{2}[ & \left(u_{\alpha \alpha}+2 u_{\alpha} \rho_{\alpha}\right) \sigma_{\alpha \alpha}+\left(u_{\alpha \beta}+2 u_{\alpha} \rho_{\beta}\right) \sigma_{\alpha \beta} \\
& +\left(u_{\beta \alpha}+2 u_{\beta} \rho_{\alpha}\right) \sigma_{\beta \alpha}+\left(u_{\beta \beta}+2 u_{\beta} \rho_{\beta}\right) \sigma_{\beta \beta} \\
& +\left(u_{\alpha} \sigma_{\alpha \alpha}+u_{\beta} \sigma_{\alpha \beta}\right)\left(L_{\alpha \alpha \alpha} \sigma_{\alpha \alpha}+L_{\alpha \beta \alpha} \sigma_{\alpha \beta}+L_{\beta \alpha \alpha} \sigma_{\beta \alpha}+L_{\beta \beta \alpha} \sigma_{\beta \beta}\right) \\
& \left.+\left(u_{\alpha} \sigma_{\beta \alpha}+u_{\beta} \sigma_{\beta \beta}\right)\left(L_{\alpha \alpha \beta} \sigma_{\alpha \alpha}+L_{\alpha \beta \beta} \sigma_{\alpha \beta}+L_{\beta \alpha \beta} \sigma_{\beta \alpha}+L_{\beta \beta \beta} \sigma_{\beta \beta}\right)\right]
\end{aligned}
$$

All functions of the right-hand side of Equation (5) are to be evaluated at the MLE $\hat{u}$.

For our setup, the joint prior distribution is $\pi\left(\lambda_{1}, \lambda_{2}\right)=\lambda_{1}^{b-1} \lambda_{2}^{d-1} e^{-\left(c \lambda_{1}+s \lambda_{2}\right)}$ and

$$
\rho\left(\lambda_{1}, \lambda_{2}\right)=\log \left[\pi\left(\lambda_{1}, \lambda_{2}\right)\right]=(b-1) \log \lambda_{1}+(d-1) \log \lambda_{2}-\left(c \lambda_{1}+s \lambda_{2}\right)
$$

which give out

$$
\begin{gathered}
\rho_{\lambda_{1}}=\frac{d \rho}{d \lambda_{1}}=\frac{b-1}{\lambda_{1}}-c, \quad \rho_{\lambda_{2}}=\frac{d \rho}{d \lambda_{2}}=\frac{d-1}{\lambda_{2}}-s, \\
u_{\alpha}=\frac{d u}{d \alpha}, \quad u_{\beta}=\frac{d u}{d \beta} \quad u_{\alpha \beta}=\frac{d^{2} u}{d \alpha d \beta}, \quad u_{\beta \alpha}=\frac{d^{2} u}{d \beta d \alpha}, \\
u_{\alpha \alpha}=\frac{d^{2} u}{d \alpha d \alpha}, \quad u_{\beta \beta}=\frac{d^{2} u}{d \beta d \beta}
\end{gathered}
$$


When

$$
\begin{aligned}
u= & \lambda_{1}, \quad u_{\lambda_{1}}=1, \quad u_{\lambda_{1} \lambda_{1}}=0=u_{\lambda_{2}}=u_{\lambda_{1} \lambda_{2}}=u_{\lambda_{2} \lambda_{1}}=u_{\lambda_{2} \lambda_{2}} \\
& \hat{\lambda}_{1 \text { Lindley }}=\hat{\lambda_{1}}+\frac{1}{2}\left[2 \rho_{\lambda_{1}} \sigma_{\lambda_{1} \lambda_{1}}+2 \rho_{\lambda_{2}} \sigma_{\lambda_{1} \lambda_{2}}+\sigma_{\lambda_{1} \lambda_{1}}\left(L_{\lambda_{1} \lambda_{1} \lambda_{1}} \sigma_{\lambda_{1} \lambda_{1}}\right.\right. \\
& \left.+L_{\lambda_{1} \lambda_{2} \lambda_{1}} \sigma_{\lambda_{1} \lambda_{2}}+L_{\lambda_{2} \lambda_{1} \lambda_{1}} \sigma_{\lambda_{2} \lambda_{1}}+L_{\lambda_{2} \lambda_{2} \lambda_{1}} \sigma_{\lambda_{2} \lambda_{1}}\right) \\
& +\sigma_{\lambda_{2} \lambda_{1}}\left(L_{\lambda_{1} \lambda_{1} \lambda_{2}} \sigma_{\lambda_{1} \lambda_{1}}+L_{\lambda_{1} \lambda_{1} \lambda_{2}} \sigma_{\lambda_{1} \lambda_{2}}\right. \\
& \left.+L_{\lambda_{2} \lambda_{1} \lambda_{2}} \sigma_{\lambda_{2} \lambda_{1}}+L_{\lambda_{2} \lambda_{2} \lambda_{2}} \sigma_{\lambda_{2} \lambda_{2}}\right]
\end{aligned}
$$

and when

$$
\begin{aligned}
u= & \lambda_{2}, \quad u_{\lambda_{2}}=1, \quad u_{\lambda_{2} \lambda_{2}}=0=u_{\lambda_{1}}=u_{\lambda_{1} \lambda_{1}}=u_{\lambda_{2} \lambda_{1}}=u_{\lambda_{1} \lambda_{1}} \\
& \hat{\lambda}_{\text {Lindley }}=\hat{\lambda_{2}}+\frac{1}{2}\left[2 \rho_{\lambda_{1}} \sigma_{\lambda_{2} \lambda_{1}}+2 \rho_{\lambda_{2}} \sigma_{\lambda_{2} \lambda_{2}}+\sigma_{\lambda_{1} \lambda_{2}}\left(L_{\lambda_{1} \lambda_{1} \lambda_{1}} \sigma_{\lambda_{1} \lambda_{1}}\right.\right. \\
& \left.+L_{\lambda_{1} \lambda_{2} \lambda_{1}} \sigma_{\lambda_{1} \lambda_{2}}+L_{\lambda_{2} \lambda_{1} \lambda_{1}} \sigma_{\lambda_{2} \lambda_{1}}+L_{\lambda_{2} \lambda_{2} \lambda_{1}} \sigma_{\lambda_{2} \lambda_{2}}\right) \\
& +\sigma_{\lambda_{2} \lambda_{2}}\left(L_{\lambda_{1} \lambda_{1} \lambda_{2}} \sigma_{\lambda_{1} \lambda_{1}}+L_{\lambda_{1} \lambda_{2} \lambda_{2}} \sigma_{\lambda_{1} \lambda_{2}}\right. \\
& \left.+L_{\lambda_{2} \lambda_{1} \lambda_{2}} \sigma_{\lambda_{2} \lambda_{1}}+L_{\lambda_{2} \lambda_{2} \lambda_{2}} \sigma_{\lambda_{2} \lambda_{2}}\right]
\end{aligned}
$$

In the above expressions $\sigma_{i j}=(i, j)$ th element in the inverse of the negative Hessian matrix, $i, j=\alpha, \beta$, and $L_{i j k}$ implies the term obtained from differentiating $\log \mathrm{L}$ with respect to $\mathrm{i}, \mathrm{j}$ and $\mathrm{k}$.

\section{References}

1. Alizadeh M, Altun E, Cordeiro GM, Rasekhi M (2018) The odd power cauchy family of distributions: properties, regression models and applications. J Stat Comput Simul 88(4):785-807

2. Alzaatreh A, Lee C, Famoye F (2013) A new method for generating families of continuous distributions. Metron 71(1):63-79

3. Aarset MV (1987) How to identify a bathtub hazard rate. IEEE Trans Reliab 36(1):106-108

4. Cordeiro GM, Alizadeh M, Ozel G, Hosseini B, Ortega EMM, Altun E (2017) The generalized odd log-logistic family of distributions: properties, regression models and applications. J Stat Comput Simul 87(5):908-932

5. Efron B, Tibshirani RJ (1994) An introduction to the bootstrap. CRC Press, Boca Raton

6. Kharazmi O, Saadatinik A (2016) Hyperbolic cosine-F families of distributions with an application to exponential distribution. Gazi Univ J Sci 29(4):811829

7. Kharazmi O, Saadatinik A, Alizadeh M, Hamedani GG (2018) Odd hyperbolic cosine-FG (OHC-FG) family of lifetime distributions. J Stat Theory Appl (JSTA) (accepted)

8. Murthy DP, Xie M, Jiang R (2004) Weibull models, vol 505. Wiley, Hoboken

9. Ramos MW, Marinho PR, Silva RV, Cordeiro GM (2013) The exponentiated Lomax Poisson distribution with an application to lifetime data. Adv Appl Stat 34(2):107-135

Publisher's Note Springer Nature remains neutral with regard to jurisdictional claims in published maps and institutional affiliations. 